\title{
APPLICATION OF STATISTICAL PROCESS CONTROL (SPC) IN THE MANUFACTURING OF MEDICAL ACCELERATORS.
}

\author{
S. M. Hanna*, Siemens Medical Solutions USA, Inc., Oncology Care Systems Group
}

\author{
Concord, CA 94520, USA
}

\begin{abstract}
Most radiation therapy machines are based on microwave linear accelerators. Manufacturing reliable accelerators and hence, reliable radiation therapy machines requires accurate, efficient, and well-controlled processes throughout the steps of electron accelerator manufacturing. Statistical Process Control (SPC) techniques are utilized at Siemens - Oncology Care Systems (OCS) to meet these requirements. SPC is routinely used to monitor different accelerator manufacturing processes. Analysis of the resulting data is used to initiate actions to preserve consistent and highquality performance. This results in processes that are stable and predictable at both higher effective capacity and lower cost. In addition, it is expected that high-energy physics projects such as the Next Linear Collider (NLC) should benefit from these techniques. Quality control techniques similar to those used by Siemens are essential to the fabrication of the large number of cavities and accelerator sections in an industrial environment.
\end{abstract}

\section{QUALITY ASSURANCE IN THE MANUFACTURING OF MEDICAL DEVICES}

Consistent high-quality and defect-free production is a clear requirement in medical devices. To achieve this requirement, medical device manufacturers usually employ Quality Management Systems (QMS) to maintain quality. Additionally, QMS help medical device manufacturers meet various regulatory quality standards such as the ISO 9001 (as a generic quality system standard) and the ISO 13485 (as a quality system standard for medical devices). In recent years, Statistical Quality Control (SQC) and its subset the Statistical Process Control (SPC), [1-3] have proven effective in improving and maintaining the quality of the medical device industry and supporting the FDA's good manufacturing practices.

\section{STATISTICAL QUALITY CONTROL}

Statistical Quality Control (SQC) is a scientific method to analyze manufacturing data. Based on this analysis, measures are taken to maintain the quality of the manufactured product. One of the techniques that are used to monitor manufacturing processes and provide feedback is Statistical Process Control (SPC). The feedback is used

*E-mail: samy.hanna@siemens.com. to maintain and improve the capability of the process and to ensure product conformance. SPC is used to control the process by signaling when adjustments may be necessary. Some techniques associated with SPC include frequency histograms and control charts. A control chart is the tool used to monitor the variation in a process and ensure that the process is in a state of control. This allows the operator to monitor the trends occurring in the process. The control chart reflects the specification limits, namely, the Upper Specification Limit (USL) and the Lower Specification Limit (LSL). In addition, it has upper and lower control limits that lie within the specification limits. The Upper Control Limit (UCL) and the Lower Control Limit (LCL) are determined by evaluating the dispersion (variability) in process, see Fig. 1. In a well-controlled process, these limits can be chosen to be equal to $\mu \pm \mathbf{3 \sigma}$ respectively, where $\sigma$ is the process standard deviation and $\mu$ is the process mean. These statistical limits are normally called the " 3 sigma control limits". In a normal (Gaussian) distribution, $99.73 \%$ of the values measured lie in interval of width $6 \sigma$.

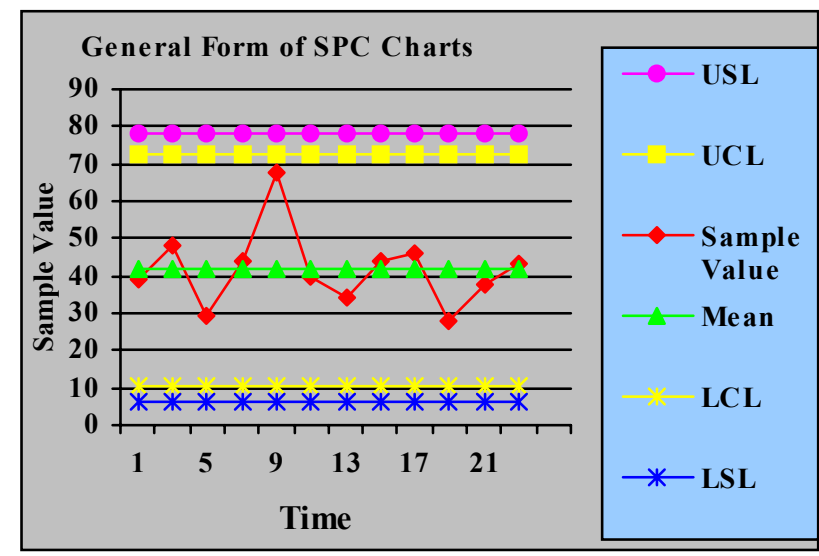

Figure 1: Typical SPC chart.

\section{APPLICATIONS OF SPC IN THE MANUFACTURING OF SIEMENS MEDICAL ACCELERATORS}

At Siemens Medical Solutions USA, Inc., Oncology Care Systems Group, we have been implementing various Computer-Aided Processes (CAP) in several critical areas of the accelerator manufacturing. The CAP approach provides accurate and efficient techniques to characterize accelerator structures [4-6]. These techniques are implemented at different phases of development and of 
manufacturing of the accelerators. These CAP techniques lend themselves to the use of SPC. The data is analyzed and displayed graphically a control chart, from which one can analyze variations in the process. Based on these control charts, the processing engineer can decide on the stability of the process as well as causes of deviations. The role of SPC is depicted in Fig. 2, below.

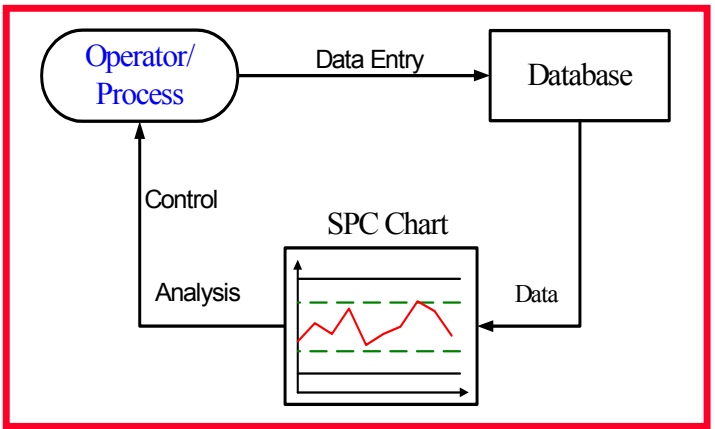

Fig. 2 Role of SPC in controlling different accelerator manufacturing process .

Each manufacturing process is characterized by a set of measurable parameters. These parameters, tables, and plots are transmitted to a database where they are stored on the network. The flow of data and documents used in accelerator manufacturing process at Siemens-OCS is shown schematically in Fig. 3, below.

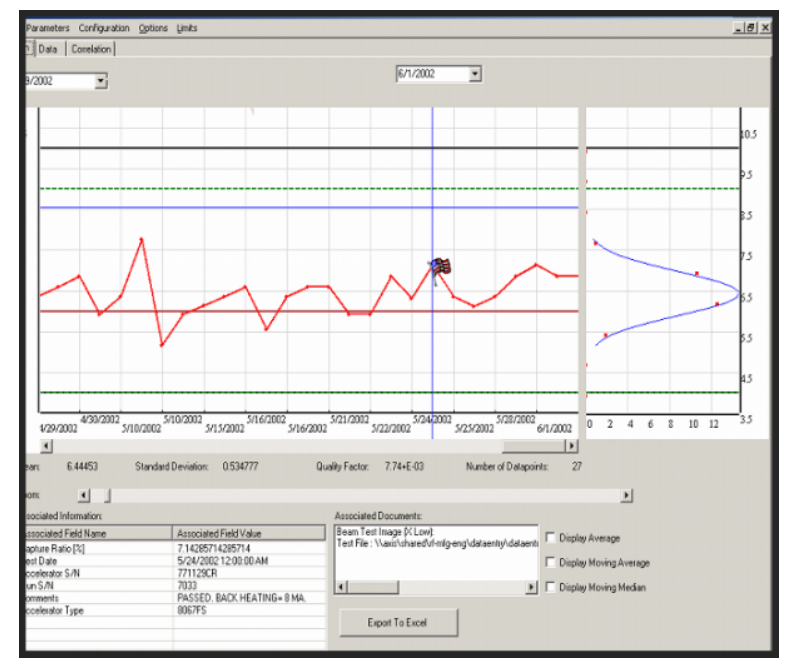

Figure 4: SPC chart of a parameter characterizing a linear accelerator at Siemens-OCS.

Our operators are trained to recognize trends occurring in the process by observing the SPC charts such as the one shown in Fig. 4. They are also trained to correct the conditions before the process approaches one of its limits. By being alert to these trends and acting accordingly and promptly, consistent process output is achieved. Ultimately, higher yield would result as the process is guarded from approaching its limits and fewer parts are out of their engineering specifications.

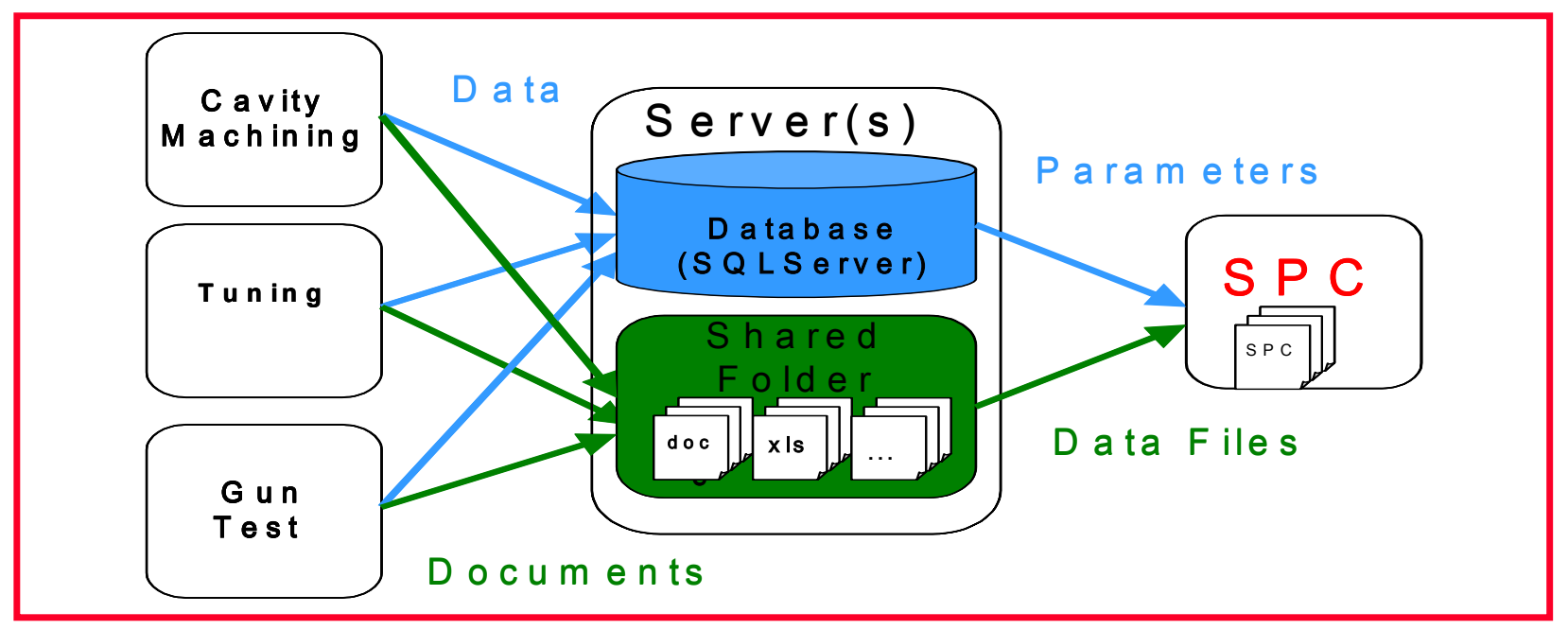

Figure 3 Flow of data and documentation to the database and the SPC system.

We are also using the approach depicted in Fig. 3 to archive data records resulting from each manufacturing process. On the resulting SPC chart one can use the curser to select a data point. A summary of related parameters are instantly displayed. If further information is needed, the full record (a table or a plot) can be retrieved from the server on the network. The example displayed in Fig. 4, shows some of data retrieval capability of our approach.
As shown in Fig. 4, the overall performance of the process over long periods of time is displayed as a frequency histogram and a fitting distribution. Our process engineers use these resulting distributions to verify the effect of the measures taken to tighten the process limits and hence improve consistency even further. 


\section{SPC AS A FEEDBACK TOOL FOR ENGINEERING DESIGN}

In designing a linear accelerator, many constraints are to be considered and satisfied. Some of these constraints have competing, or even conflicting, Therefore, trade-offs are usually needed. Our processing engineers use SPC tools to analyze the ultimate limits of different manufacturing processes. Results of these analyses and supporting data are then shared with the accelerator design engineers. This feedback on the process capabilities can provide the design engineer with information needed to tighten some tolerances related to the manufacturing process. This would allow the relief of other competing design constraints and still meet manufacturing requirements. Ultimately, a better design that is efficiently manufactured is achieved. Fig. 5 depicts the role of SPC in the design cycle.

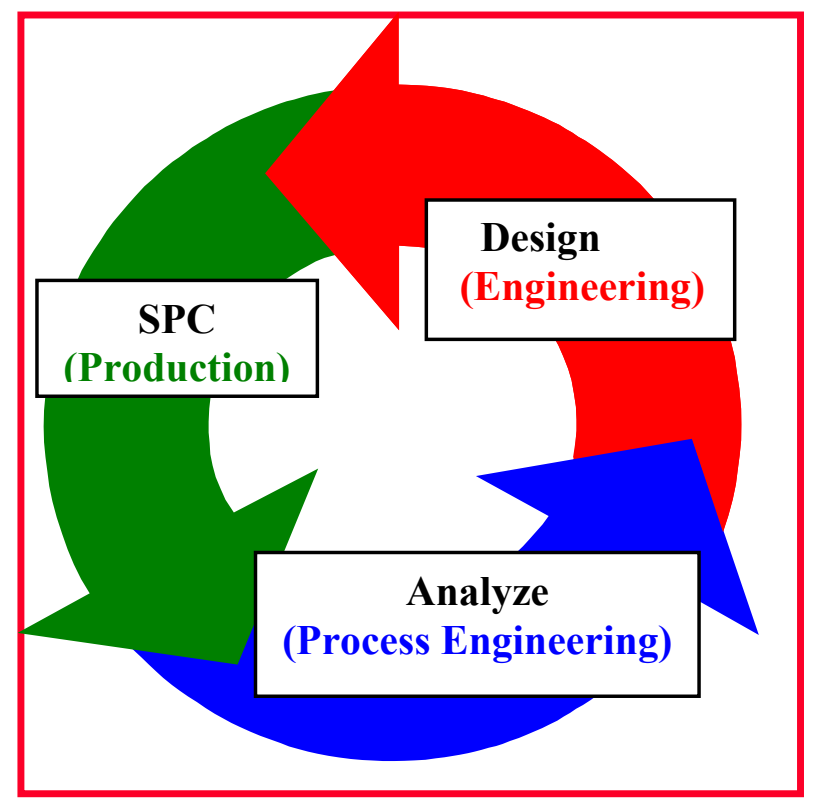

Fig. 5 Role of SPC in the design cycle.

\section{APPLICATIONS OF SPC IN PARTNERSHIPS WITH SUPPLIERS}

A strengthening factor in the partnerships with our suppliers is providing feedback information on the quality of their supplied components in a timely manner. Our SPC tools have been effective in compiling data measured by our suppliers as well as our processes and analyzing them. Reports resulting from these analyses and the associated data are shared with the relevant suppliers.

\section{ACKNOWLEDGMENT}

Thanks to Shawn Hilton for his useful input and suggestions that were essential to the development of the SPC tool. The Programming skills of Daniel Grossmann and Anselm Vossen made that development possible.

\section{REFERENCES}

[1] D. Raheja, “ Assurance Technologies: Principles and Practices ", McGraw Hill, Inc., 1991.

[2] J. Heizer and B. Render, "Operations Management", 6th Ed., Prentice Hall, 2001

[3] R. T. Amsden, H. E. Butler, and D. M. Amsden, “ SPC Simplified: practical Steps to Quality", Productivity, Inc., 1998.

[4] S. M. Hanna, "Development of Characterization Techniques for X-Band Accelerator Structures," Proc. Of the 2000 European Particle Accelerator Conference, Vienna, Austria, pp 2521-2523, 2000.

[5] S .M. Hanna, et al., "Automated System for Conditioning A Linear Accelerator", US Patent No. 6483263, Issued November 2002.

[6] S .M. Hanna, et al., "Computer-Aided Tuning of Charged Particle Accelerators ", US Patent No. 6498444B1, Issued December 2002. 